\title{
Decreased glycolytic metabolism contributes to but is not the inducer of apoptosis following IL-3-starvation
}

S Gonin-Giraud ${ }^{1,2}$, A-L Mathieu ${ }^{1,2}$, S Diocou ${ }^{1}$, M Tomkowiak $^{1}$, G Delorme ${ }^{1}$ and J Marvel ${ }^{*, 1}$

1 Immuno-Apoptose, INSERM U503, Centre d'études et de Recherches en Virologie et en Immunologie, 21 avenue Tony Garnier, 69365 Lyon cedex 07, France

2 These two authors contributed equally to the work.

* Corresponding author Jacqueline Marvel, Equipe Immuno-Apoptose, INSERM U503, CERVI, 21, avenue Tony Garnier, 69365 Lyon cedex 07, France. Tel: 33437282 350; Fax: 33437282 341;

E-mail: marvel@cervi-lyon.inserm.fr

Received 27.11.01; revised 9.4.02; accepted 14.5.02 Edited by RA Knight

\section{Abstract}

IL-3 regulates the glycolytic pathway. In Baf-3 cells IL-3 starvation leads to a decrease in glucose uptake and in lactate production. To determine if there is a link between the decreased metabolism induced by growth factor-starvation and the induction of cell death, we have compared the cell death characteristics and the metabolic modifications induced by IL-3-deprivation or glucose-deprivation in Baf-3 cells. We show that in both conditions cells die by an apoptotic process which involves the activation of similar Caspases. Different metabolic parameters (i.e. intracellular ATP levels and lactate accumulation in the culture medium) were measured. We show that IL-3 deprivation leads to a partial decrease in lactate production in contrast to glucose deprivation that completely inhibits lactate production. Similarly following IL-3-starvation a significant drop in the intracellular ATP levels in live cells is observed only after $16 \mathrm{~h}$ when a large fraction, more than 50 per cent of cells, is already apoptotic. On the contrary, glucose deprivation is followed by an abrupt decrease in ATP levels in the first $2 \mathrm{~h}$ of treatment. However, in the presence of IL-3, cells are able to survive for an extended time in these conditions since $70 \%$ of cells survived with low ATP levels for up to $16 \mathrm{~h}$. This was not due to partial inhibition of the apoptotic process by the low level of ATP as glucose-deprivation in the absence of IL-3 led to faster death kinetics of Baf-3 cells compared with IL-3 starvation only. These results indicate that the drop in ATP levels and the triggering of apoptosis can be dissociated in time and that when the glycolytic pathway is strongly inhibited, cells are able to survive with relatively low ATP levels if IL-3 is present. Finally we show that induction of bcl-x by IL-3 protects cells from glucose-deprivation induced cell death.

Cell Death and Differentiation (2002) 9, 1147-1157. doi:10.1038/sj.cdd.4401079

Keywords: glycolysis; apoptosis; PI3-kinase; Akt; Bcl-x
Abbreviations: IL-3, interleukin-3; GSK-3, glycogen synthase-3 kinase; 2-DG, 2-deoxy-D-glucose

\section{Introduction}

There is increasing evidence that cellular survival is dependent upon growth factor availability that inhibits intrinsic programmed cell death. These factors are also necessary to promote cellular proliferation and metabolism activation. Interleukin-3 (IL-3) inhibits apoptosis through the activation of different signalling pathways. ${ }^{1-3}$ Among them, the PI3kinase/Akt pathway has been shown to be essential in mediating cellular survival. ${ }^{2,4,5}$ Following a PI3-kinase dependent activation, Akt phosphorylates and inhibits proapoptotic proteins like Bad, Caspase-9 and Forkhead family transcription factors. ${ }^{4,6,7}$ In addition to its involvement in regulating cellular survival, Akt has also been shown to mediate the effects of insulin on cellular metabolism. In particular, Akt stimulates glucose uptake through the activation of the glucose transporters GLUT1 and GLUT4. Indeed, a constitutively active form of Akt increases GLUT1 mRNA and protein accumulation in a mouse hepatoma cell line. ${ }^{8}$ Akt can also induce GLUT4 translocation to the plasma membrane in adipocytes, increasing glucose uptake by these cells. ${ }^{9}$ One of the first Akt targets that has been identified is the glycogen synthase kinase-3 (GSK-3), which plays a role in glycogen synthesis by inhibition of glycogen synthase in unstimulated cells. ${ }^{10}$ Akt has been shown to phosphorylate, and therefore inhibit GSK-3, allowing glycogen storage.

Recent evidence suggests that Akt could mediate cellular survival in part through glycolytic metabolism activation. Indeed, some Akt substrates regulating the glycolytic metabolism are involved in the control of cellular survival. For example, it has been shown that overexpression of active GSK-3 in Rat-1 or PC12 cells induces apoptosis. ${ }^{11}$ In these cells, transfection of a dominant negative form of GSK-3 was sufficient to protect against apoptosis induced by PI3-kinase inhibition. Similarly, GSK3 specific inhibition maintains neuron survival in the presence of the PI3-kinase inhibitor Ly 294002. ${ }^{12}$ More recently, hexokinase that is involved in coupling intramitochondrial ATP synthesis to glucose metabolism has been shown to be a downstream target for Akt. Transfection of Rat-1 cells with hexokinase protected against apoptosis induced by growth factor withdrawal and UV treatment in a glucose dependent manner, therefore mimicking the effects of Akt. ${ }^{13}$

These results suggest that glycolytic metabolism activation may be an essential pathway activated by growth factors that leads to cellular survival. ${ }^{14}$ Constitutive glycolytic metabolism activation may be sufficient to protect cells against various apoptotic stimuli. This is consistent with the fact that anaerobic glycolysis is frequently 
increased in tumour cell lines. ${ }^{15}$ In IL-3 dependent cells, stable transfection of the $\mathrm{v}$-abl kinase allows survival in the absence of growth factor and stimulates glucose transport. ${ }^{14}$ However, this survival is strongly decreased in the presence of glucose metabolism inhibitors, demonstrating the importance of glycolytic metabolism activation in the suppression of apoptosis. More recently, it was suggested that a key event in apoptosis inhibition by growth factors is the promotion of nutrients uptake and cellular metabolism. ${ }^{16}$ Indeed growth factor withdrawal leads to a decrease in cell size that is accompanied by a decrease in intracellular ATP concentration and mitochondrial membrane potential. Low glucose concentrations in the medium prevented the ability of growth factors to induce cellular survival, suggesting that part of the anti-apoptotic signalling pathway delivered by growth factors is dependent upon glycolytic metabolism activation. Similarly, GLUT1 overexpression in IL-3 dependent cells delayed apoptosis induced by IL-3 starvation, reinforcing the idea that metabolism activation is essential in promoting cellular survival. ${ }^{17}$

To better understand the link between metabolism and apoptosis inhibition, we have characterised cell death induced by growth factor or glucose starvation in the IL-3 dependent Baf-3 cell line. We show that both death stimuli lead to an apoptotic process involving similar Caspases. In the absence of IL-3 stimulation, we observed a partial decrease of lactate production and intracellular ATP concentration. When glycolysis is inhibited in these cells, lactate production is completely stopped and there is an abrupt decrease of intracellular ATP concentration. However, Baf-3 apoptosis is faster following IL-3 starvation than following glucose withdrawal, suggesting that the decreased metabolism is not the principal triggering event leading to the activation of the death machinery. We also show that $b c /-x$ induction, but not Akt activation, can protect Baf-3 cells from glucose starvation induced cell death.

\section{Results}

\section{IL-3 or glucose deprivation induce a similar pattern of apoptotic cell death}

In haematopoietic cell lines IL-3 regulates the glycolytic metabolism. ${ }^{14,18,19}$ We have previously shown that in a mutant Baf-3 clone, constitutive glycolytic metabolism was associated with cell survival in the absence of IL-3. ${ }^{20}$ In order to define if the decreased glycolytic metabolism observed following IL-3 removal was sufficient for the induction of apoptosis we have studied the consequence of glucose deprivation on Baf-3 cell survival. Baf-3 cells were grown in DMEM without glucose but supplemented with FCS and WEHI supernatant resulting in an estimated glucose concentration of $250 \mathrm{mg} /$ litre. This medium is hereafter called low glucose medium. Results in Figure $1 \mathrm{~A}$ show that in low glucose medium cells start dying after $60 \mathrm{~h}$ culture in the presence of IL-3. Dead cells show a positive staining by annexin $\mathrm{V}$ (Figure 1B), suggesting that they died by an apoptotic pathway. This was confirmed by measuring caspase activity at different time points following IL-3 removal or transfer to low glucose medium. The activity of caspases 3 , 7 and 8 (Figure 1C) and caspases 6 and 8 (Figure 1D) were measured using the fluorogenic substrates DEVD-AMC and VEID-AMC, respectively. These are the main caspases activated following IL-3 withdrawal with a maximal activity observed $10 \mathrm{~h}$ after IL-3 starvation (Figure 1C,D, left panels) and reference. ${ }^{21}$ When Baf-3 cells are cultured in low glucose medium similar caspase activities are detected with a maximal value $50 \mathrm{~h}$ after transfer to low glucose medium (Figure 1C,D, right panels). The cleavage of two other substrates (YVAD and WEHD) recognised by caspases 1, 4, 5 and 11 was also measured but no significant activity was detected whether cells were grown in IL-3 depleted medium or low glucose medium (data not shown). These results indicate that in Baf-3 cells, glucose starvation in the presence of IL-3 leads to apoptosis and suggest that similar caspases are activated following glucose or IL-3 deprivation.

The kinetics of cell death observed when Baf-3 cells were grown in low glucose medium was much slower than it was when IL-3 was removed from the culture. These results suggest that Baf-3 cells are more sensitive to IL-3 than to glucose starvation. However the low level of glucose present in the culture medium could be sufficient to maintain the glycolytic metabolism of Baf-3 cells. Therefore we have compared Baf-3 cell survival in low glucose medium containing the glucose analogue 2-deoxyD-glucose, which is a potent inhibitor of glycolysis, to survival in the absence of IL-3. In the presence of 2-deoxyD-glucose, similarly to what was found when Baf-3 cells were grown in low glucose medium, cell death was associated with Caspase 3 activation indicating that it was an apoptotic process (Figure $2 \mathrm{~A}$ ). This death process was rapid as single cells simultaneously acquired early and late hallmarks of apoptosis. Indeed, a similar increase in the percentages of propidium iodide, Annexin $\mathrm{V}$ and Caspase 3 positive cells was found at various time points for the different culture conditions (Figure 2B). Results in Figure $2 \mathrm{C}$ show that when Baf-3 cells are cultured in low glucose medium containing $\mathrm{IL}-3$ in the presence of 2deoxy-D-glucose, cell death occurs much earlier than when cells are cultured in low glucose medium only (Figure 1A). Indeed in low glucose medium it takes an average of $60 \mathrm{~h}$ to reach $50 \%$ of dead cells in the culture compared to $18 \mathrm{~h}$ when 2-deoxy-D-glucose is added to the culture. However the death kinetics of Baf-3 cells starved only of IL-3 is still significantly faster (Figure $2 \mathrm{C}$ ). This could indicate that although glucose deprivation leads to apoptosis of Baf-3 cells, the decreased metabolism which has been described following IL-3 deprivation is not the main pathway responsible for the triggering of the apoptotic process induced by growth factor starvation.

\section{The decreased glycolytic metabolism induced by IL-3 starvation is not sufficient to trigger apoptosis}

To test this hypothesis we have compared the glycolytic metabolism of Baf- 3 cells cultured in the absence of IL-3 or in the presence of IL-3 in low glucose medium containing 2deoxy-D-glucose. We have previously shown that Baf-3 cells 
A

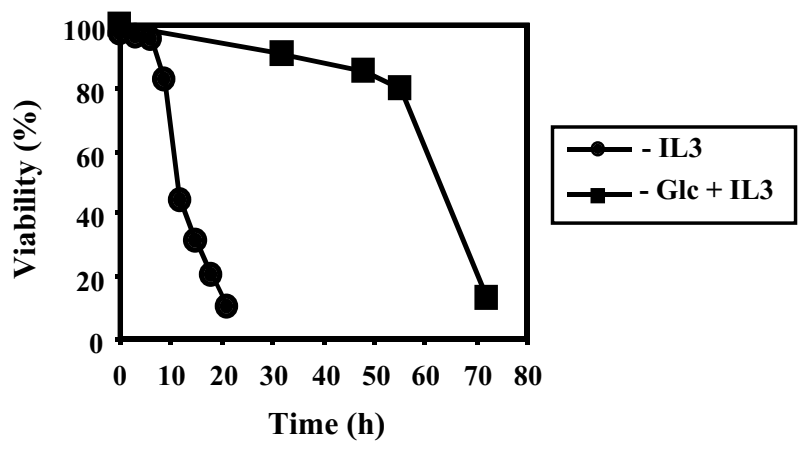

C

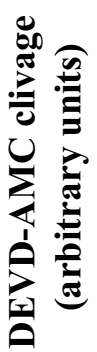

D

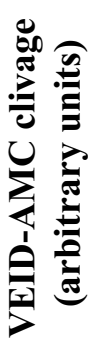

- IL3

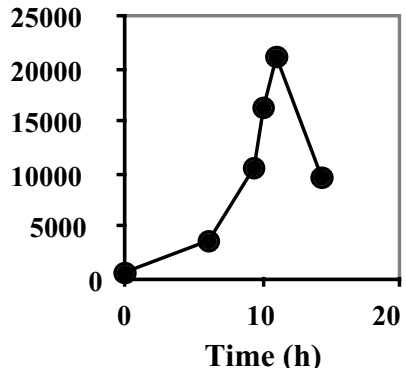

- IL3

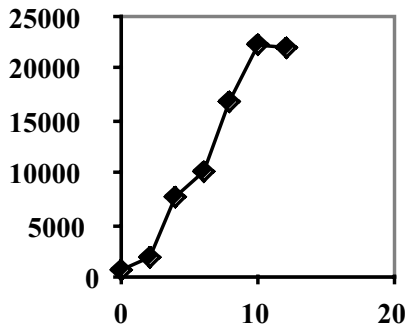

Time (h)
B

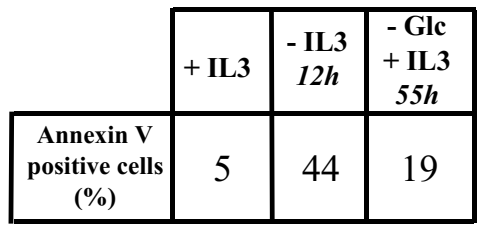

- Glc + IL3

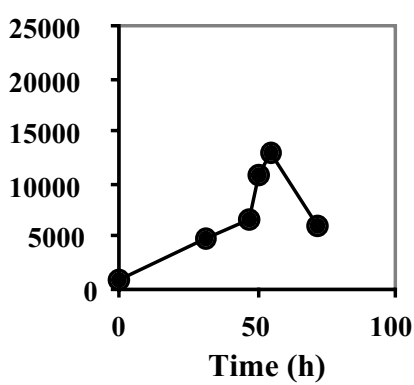

- Glc + IL3

Figure 1 Baf-3 cells undergo an apoptotic process following IL-3 starvation or glucose deprivation. (A) Baf-3 cells were cultured in the absence of IL-3 (-IL3) or in a low glucose medium containing IL-3 (-GIC+IL3). Viability was assessed at different time points by propidium iodide exclusion. One representative experiment out of three is presented. (B) Baf-3 cells were cultured in the presence of IL-3 (+IL3), in the absence of IL-3 (-IL3) or in a low glucose medium containing IL-3 (-GIC+IL3). Annexin V staining was performed after 12 or $55 \mathrm{~h}$ according to the conditions. One representative experiment out of three is presented. (C,D) Baf-3 cells were cultured in the absence of IL-3 (-IL3) or in a low glucose DMEM in the presence of IL-3 (-GlC+IL3). Caspases 3, 7 and 8 (C) and Caspases 6 and 8 (D) activities were measured in cell lysates at different time points using the fluorogenic substrates DEVD-AMC and VEID-AMC, respectively. One representative experiment out of three (C) or two (D) is represented

like other bone marrow derived cell lines mainly perform anaerobic glycolysis, i.e. most of the glucose is degraded into lactate. ${ }^{20}$ Therefore we have monitored the glycolytic activity of Baf-3 cells under different culture conditions by measuring the lactate produced in the medium. Results in Figure $3 \mathrm{~A}$ show that although lactate production by Baf-3 cells is significantly decreased in absence of IL-3, some glycolytic metabolism still takes place during the first $8 \mathrm{~h}$ of culture. In contrast when Baf-3 cells are grown in low glucose medium containing 2-deoxy-D-glucose, lactate production is completely abrogated. For cells maintained in culture, glycolysis is normally the main source of ATP. However, amino acids such as glutamine can be processed in the TCA cycle and support ATP production. Therefore we have measured the level of 
B

A

\section{+ IL3}

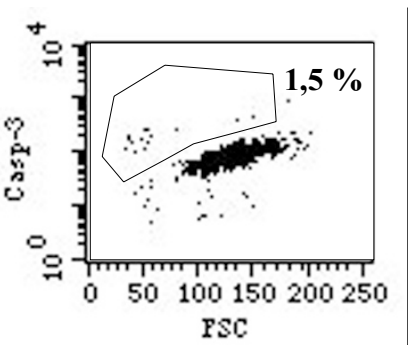

- 2DG

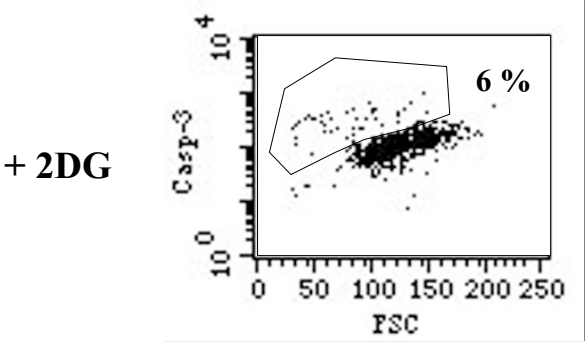

- IL3
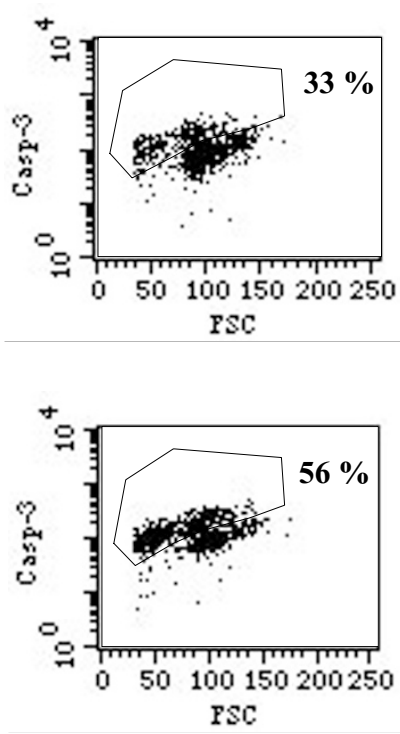
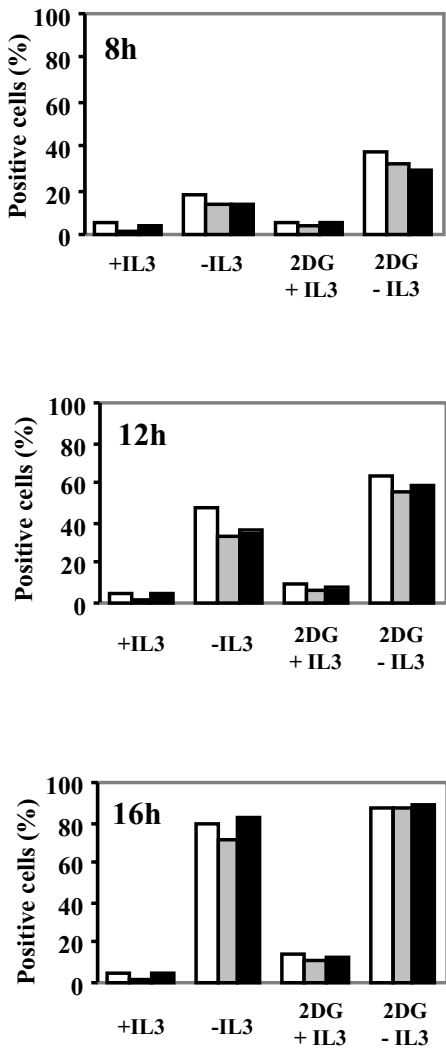

C

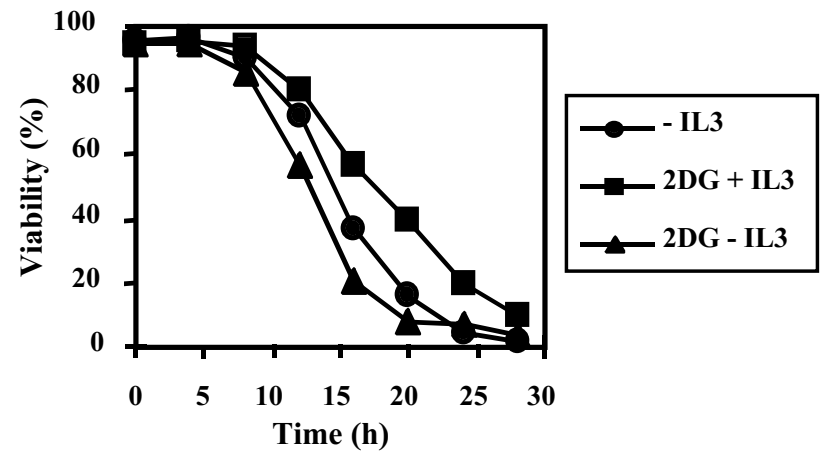

Figure 2 Baf-3 cells loss of viability is slower following glycolysis inhibition than following IL-3 starvation. (A) Baf-3 cells were cultured in the presence (left panel, +IL3) or in the absence (right panel, -IL3) of IL-3 in normal medium (upper panel, -2DG) or in low glucose medium containing 6 mM 2-deoxy-D-glucose (lower panel, $+2 \mathrm{DG}$ ). Activated Caspase 3 staining was performed after $12 \mathrm{~h}$ as described in Materials and Methods. One representative experiment out of three is presented. (B) Baf-3 cells were cultured in the presence (+IL3) or in the absence (-IL3) of IL-3 or in low glucose medium containing $6 \mathrm{mM}$ 2-deoxy-D-glucose in the presence $(2 D G+I L 3)$ or in the absence of IL-3 (2DG-IL3). Viability was assessed at different time points as indicated on the graphs using Annexin V (white bars), activated Caspase 3 (grey bars) or propidium iodide staining (black bars). One representative experiment out of three is presented. (C) Baf-3 cells were cultured in the absence of IL-3 (-IL3) or in a low glucose medium with $6 \mathrm{mM}$ 2-deoxy-D-glucose in the presence (2DG+IL3) or absence of IL-3 (2DG-IL3). Viability was assessed at different time points by propidium iodide exclusion. One representative time course out of two is presented

ATP in Baf-3 cells following IL-3 starvation or glycolysis inhibition (Figure 3B). When Baf-3 cells are deprived of IL-3 the ATP level is maintained close to its initial level during the first $8 \mathrm{~h}$ of culture. This confirms that some glycolysis still takes place in the first $8 \mathrm{~h}$ following IL-3 removal. However after $16 \mathrm{~h}$ of IL-3 depletion, the quantity of ATP contained in
$10^{6}$ live Baf-3 cells was decreased by $60 \%$ in agreement with the decreased lactate production observed at the same time. These results confirm that in absence of IL-3 glycolysis slows down leading to a decrease in lactate production and ATP levels. This effect, however, is only detectable in live cells at time points where a significant fraction of Baf-3 cells are 
already apoptotic ( $60 \%$ dead cells at $16 \mathrm{~h}$ ). In contrast when glycolysis is inhibited by 2-deoxy-D-glucose, whether IL-3 is present or not, the ATP level drops to $20 \%$ of control level in the first hours of treatment and is maintained at that level for up to $16 \mathrm{~h}$ (Figure 3B and data not shown). This confirms that the maintenance of the ATP level in Baf-3 cells is mainly dependent on glycolysis. However in these conditions it takes at least $12 \mathrm{~h}$ before the first apoptotic cells are detected. This is not due to impaired apoptosis due to low levels of ATP as Baf-3 cells cultured in low glucose medium containing 2deoxy-D-glucose in absence of IL-3 had a death kinetic which was slightly faster than Baf-3 cells only deprived of IL-3 (Figure 2C). As a whole these results indicate first that the drop in ATP observed following IL-3 removal is unlikely to be the only apoptosis triggering event, second that IL-3 is able to delay the apoptosis resulting from glycolysis inhibition.

\section{Inhibition of glycolysis by 2-deoxy-D-glucose does not affect AKT activation or Bcl-X protein levels}

IL-3 regulates Baf-3 cell survival by activating the PI3-kinase/ AKT pathway and by leading to $\mathrm{Bcl}-\mathrm{X}$ up-regulation via the jak/stat and the MAP-kinase pathways. ${ }^{1-3,22}$ The drop in the intracellular ATP level induced following the inhibition of glycolysis by 2-deoxy-D-glucose could affect activation of these kinases by IL-3. Hence we have measured the phosphorylation of AKT following $8 \mathrm{~h}$ of culture in low glucose medium containing 2-deoxy-D-glucose. Results in Figure 4A show that in the presence of IL-3, the AKT phosphorylation on ser 473 was similar whether glycolysis was inhibited or not. In contrast, IL-3 starvation led to decreased AKT phosphorylation in both conditions. Similarly, in the presence of IL-3 the $b c l-x$ mRNA and protein levels were maintained for up to $8 \mathrm{~h}$

A

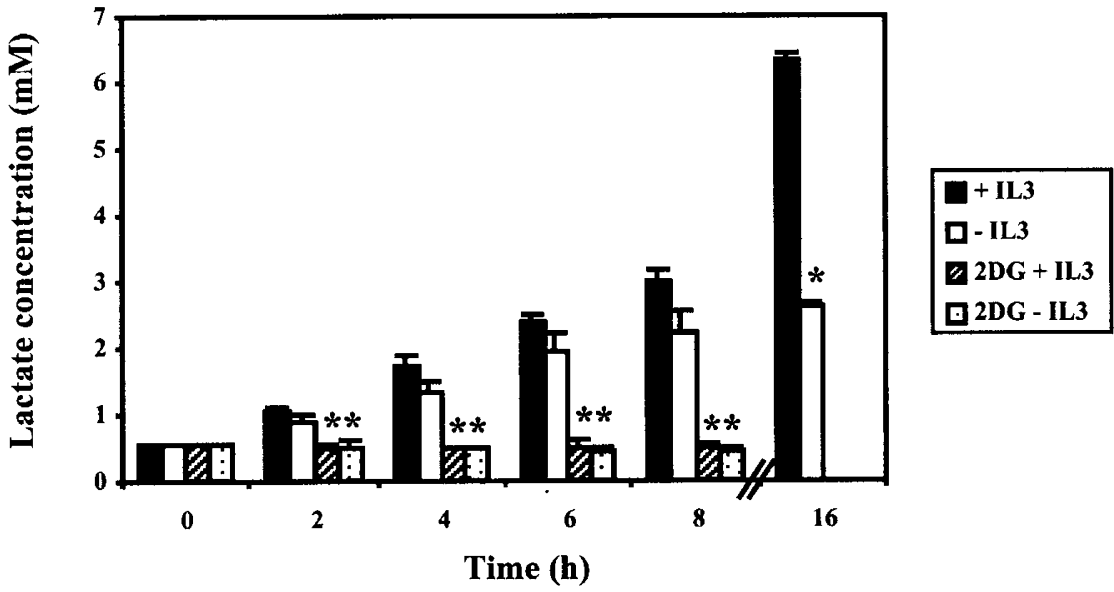

B

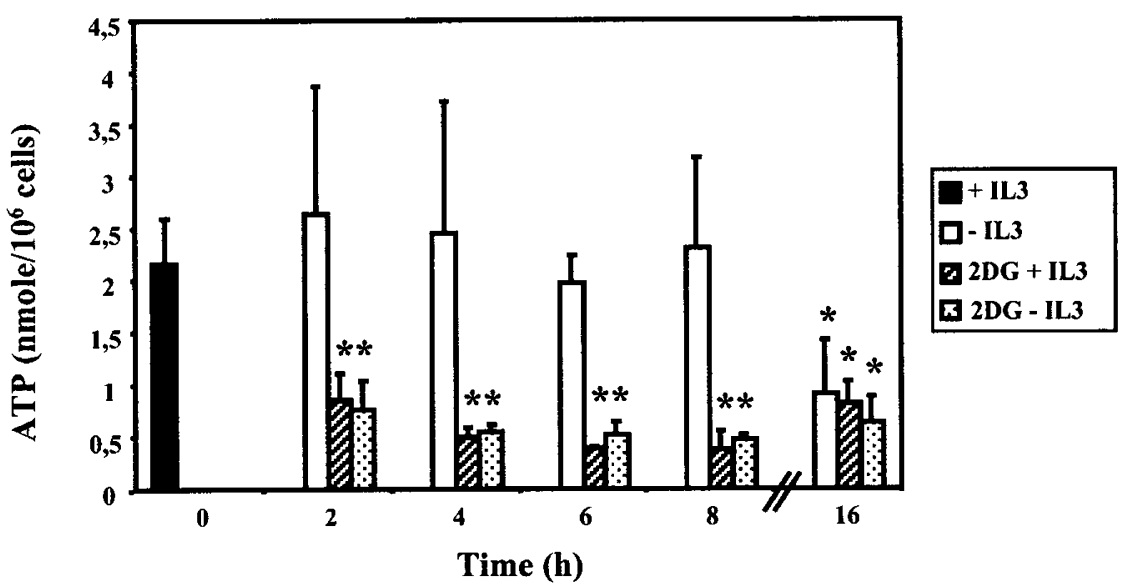

Figure 3 Lactate production and intracellular ATP levels following IL-3 starvation or glycolysis inhibition. Baf-3 cells were maintained in the presence of IL-3 (closed bars), in the absence of IL-3 (empty bars) or in a low glucose medium containing $6 \mathrm{mM}$ 2-deoxy-D-Glucose in the presence (hatched bars) or absence (dotted bars) of IL-3. (A) Lactate concentration (mmol/l) for $2 \times 10^{5}$ cells was determined every $2 \mathrm{~h}$ during $8 \mathrm{~h}$ and after $16 \mathrm{~h}$. Data are presented as mean values $+/-$ S.D. of three independent experiments. ${ }^{*}: P<0.05$ (two tailed Student's test), at different time points, lactate level values were compared to the one obtained in the presence of IL-3 at the same time point. (B) ATP content of Baf-3 cells was determined at different times by Luciferase-Luciferin method. Data are presented as mean values $+/-$ S.D. of three independent experiments. ${ }^{*}: P<0.05$ (two tailed Student's test), at different time points, ATP level values were compared to the one obtained in presence of IL-3 at time 0 
A

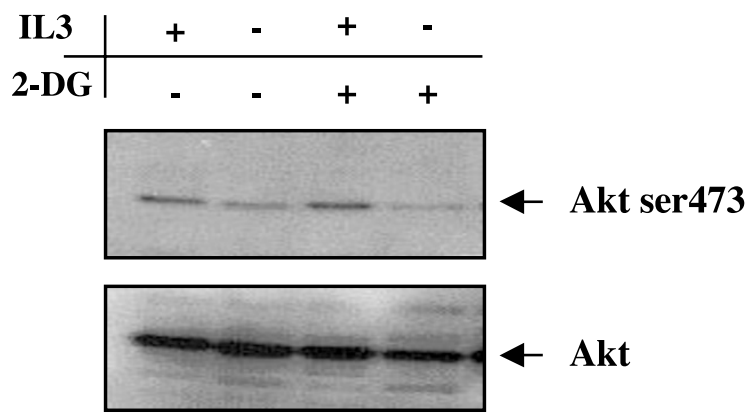

B

$\mathbf{C}$
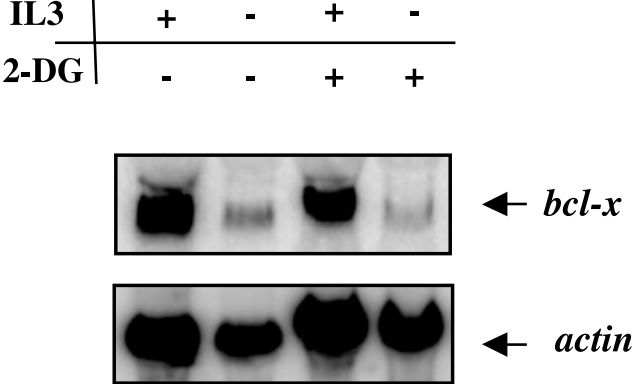
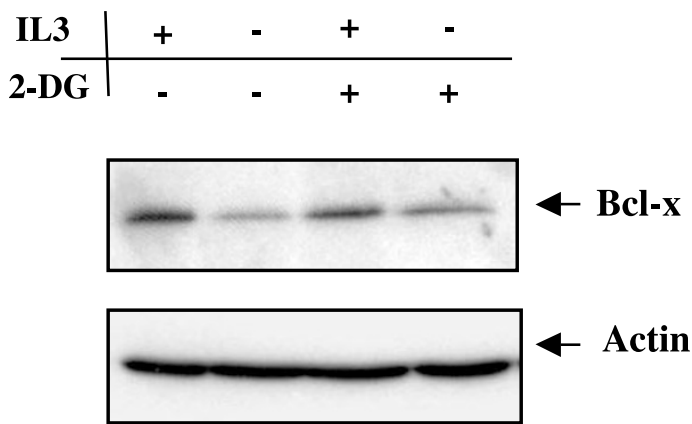

Figure 4 Glycolysis inhibition does not affect the activation of the PI3-kinase/Akt pathway or Bcl-X expression. Baf-3 cells were cultured for $8 \mathrm{~h}$ in DMEM or in a low glucose medium containing or not $6 \mathrm{mM} 2$-deoxy-D-Glucose, in the presence or in the absence of IL-3. (A) After cell lysis, $50 \mu \mathrm{g}$ of total proteins from each sample were loaded on $10 \%$ acrylamide gel. Detection of phospho-Akt or total Akt was done as described in the experimental procedure. (B) Total RNA was isolated as described in experimental procedure and $15 \mu \mathrm{g}$ were used for the Northern blot analysis using the $b c /-x$ and actin probes. (C) Cells were lysed and $50 \mu \mathrm{g}$ of total proteins were loaded on $10 \%$ acrylamide gel. Detection of $\mathrm{Bcl}-\mathrm{X}$ and Actin was done as described in the experimental procedure

following glycolysis inhibition (Figure 4B,C). These results suggest that although the intracellular ATP level is strongly decreased in the first $2 \mathrm{~h}$ following glycolysis inhibition, activation of these survival signalling pathways downstream of IL-3 is sustained. Glycolysis inhibition therefore does not trigger apoptosis by interfering with the expression of $b c /-x$ or the activation of the PI3-kinase/Akt pathway. In order to identify which one of these pathways is involved in the protection of Baf-3 cells against the apoptosis induced by glycolysis inhibition, we have studied the protection conferred to Baf-3 cells by an activated PI3-kinase/Akt pathway or by Bcl-X over-expression.

\section{The PI3-kinase/Akt pathway does not protect Baf-3 cells from apoptosis induced by glycolysis inhibition}

To investigate the role of Akt in the protection against glycolytic metabolism alteration, we have used the S4 mutant which has been obtained by retroviral insertion mutagenesis of Baf-3 cells. ${ }^{20}$ We have previously shown that these cells survive in the absence of IL-3 for several days with low $\mathrm{Bcl}-\mathrm{X}$ protein levels and that survival was dependent on the IL-3 independent PI3-kinase/Akt pathway activation. ${ }^{20}$ We first checked that inhibition of glycolysis with 2-deoxy-D-glucose did not alter the constitutive phosphorylation of Akt. Results in Figure 5A show that the Akt phosphorylation observed in the absence of IL-3 in the S4 cell line was maintained for the first $8 \mathrm{~h}$ following glycolysis inhibition. In contrast cell survival in the absence of IL-3 was strongly affected if 2-deoxy-Dglucose was added to the culture medium (Figure 5B). Similarly to parental Baf-3 cells, death was associated with early Caspase 3 activation that could be detected as early as $8 \mathrm{~h}$ following glucose starvation. These results imply that Akt is not sufficient to protect Baf-3 cells from apoptosis induced by the inhibition of the glycolytic metabolism.

\section{Constitutive bcl-x expression protects Baf-3 cells from apoptosis induced by the inhibition of the glycolytic metabolism}

We have previously shown that the expression of $b c l-x$ is induced by IL-3 in Baf-3 cells and that Bcl-X allows the survival of Baf- 3 cells in absence of IL-3. ${ }^{22,23}$ To determine if 
A

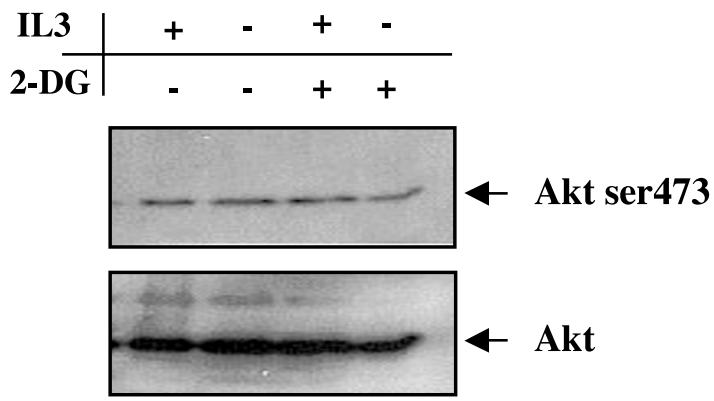

B
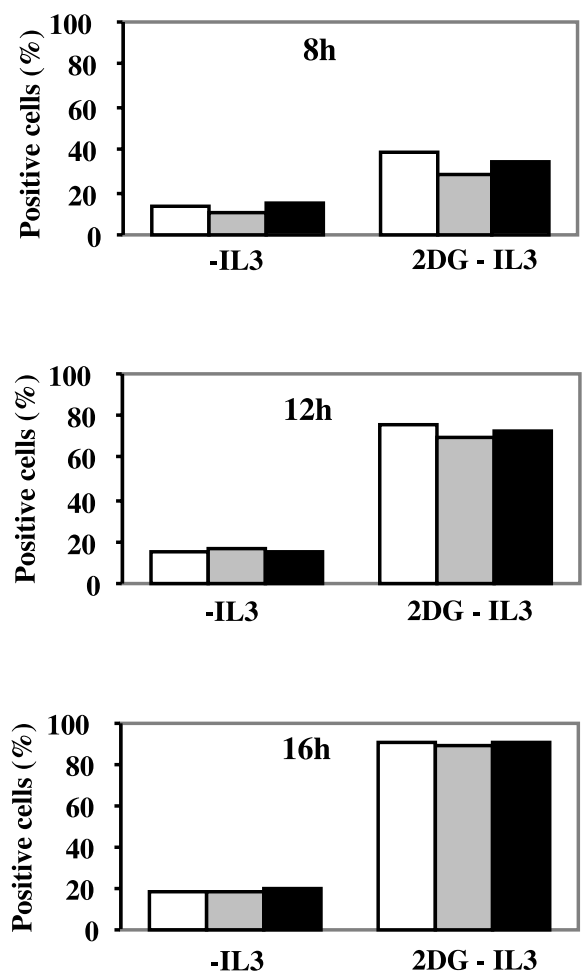

Figure 5 Akt does not protect Baf-3 cells against glycolysis inhibition. (A) S4 cells were cultured for $8 \mathrm{~h}$ in DMEM or in a low glucose medium containing $6 \mathrm{mM}$ 2-deoxy-D-Glucose, in the presence or in the absence of IL-3. After cell lysis, $50 \mu \mathrm{g}$ of total proteins from each sample were loaded on a $10 \%$ acrylamide gel. Detection of phospho-Akt or total Akt was done as described in the experimental procedure. (B) S4 cells were cultured in the absence of IL-3 in normal culture medium (-IL3) or low glucose medium containing $6 \mathrm{mM} \mathrm{2-}$ deoxy-D-Glucose (2DG-IL3). Viability was assessed at different time points as indicated on the graphs using Annexin V (white bars), activated Caspase 3 (grey bars) or propidium iodide staining (black bars). One representative experiment out of three is presented

$\mathrm{Bcl}-\mathrm{X}$ is able to protect cells against glycolytic metabolism inhibition, the viability of Baf-3 cells over-expressing Bcl-X (Baf-bcl-x) after glycolysis inhibition in absence of IL-3 was analyzed. These cells maintain a similar Bcl-X protein level when grown in the different culture conditions (Figure 6A). As shown in Figure 6B, the viability of Baf-bcl-x cells is not affected by culture in a low glucose medium containing 2 deoxy-D-glucose in the absence of IL-3 (less than $20 \%$ of apoptotic cells after $16 \mathrm{~h}$ of treatment). Therefore, the antiapoptotic protein $\mathrm{Bcl}-\mathrm{X}$ is able to protect Baf-3 cells against apoptosis induced by the inhibition of the glycolytic metabolism. However, the maintained expression of $b c l-x$ does not prevent the drop in the ATP level induced in these conditions (Figure 6C). Indeed, as observed with parental Baf-3 cells, the level of intracellular ATP was reduced by more than $70 \%$ after $2 \mathrm{~h}$ culture in a low glucose medium containing 2-deoxy-Dglucose. Thus these results suggest that the IL-3 mediated $\mathrm{Bcl}-\mathrm{X}$ induction can delay apoptosis observed when Baf-3 cells glycolysis is inhibited in the presence of IL-3. Protection by $b c l-x$, however, does not correlate with a preserved intracellular ATP level.

\section{Discussion}

In the absence of extra-cellular signals such as growth factors stimulation, cells die by apoptosis. Growth factors also regulate the glycolytic metabolism of cells thus promoting energy production. As shown in this report and in previous ones, ${ }^{18,19,24,25}$ growth factor withdrawal induces a metabolic slowdown that is revealed by a drop in glucose uptake, lactate production and in intracellular ATP concentration. IL-3 also regulates the expression of key genes involved in the control of glucose metabolism such as Glut1, hexokinase 2 or phospho-fructo kinase. ${ }^{17,26,27}$ The regulation of glucose metabolism by growth factors might be involved in the inhibition of apoptosis by these factors. Indeed, growth factor independent-survival induced by a number of oncogenes is associated with an activated glycolytic metabolism. For example, over-expression of the $\mathrm{v}$-abl kinase in an IL-3 dependent cell line leads to IL-3 independent survival and is correlated with a stimulation of glucose uptake. ${ }^{14}$ More recently it was shown that the capacity of different growth factors to promote survival of an IL-3 dependent cell line is correlated with their ability to stimulate glucose utilisation. Moreover, growth factor starvation-induced-apoptosis was delayed by over-expression of Glut 1 in these cells, suggesting that the maintenance of glucose metabolism can contribute to the inhibition of apoptosis. ${ }^{17}$

In this study we have compared cell death induced by growth factor starvation or by glucose starvation. We show that, in the Baf-3 cell line, glycolysis inhibition or IL-3 deprivation leads to an apoptotic process involving similar Caspases. Following IL-3 withdrawal, we observed a slow and partial decrease of lactate production and intracellular ATP concentration, confirming that IL-3 regulates the glycolytic metabolism of these cells. In contrast, when glycolysis is inhibited using 2-deoxy-D-glucose, there is a rapid drop in intracellular ATP concentration and in lactate production detectable as soon as $2 \mathrm{~h}$ after treatment. However, there is a more rapid loss of viability when Baf3 cells are maintained in the absence of IL- 3 than following glycolysis inhibition in the presence of IL-3 suggesting that the decreased glycolytic metabolism observed following IL3 removal is not the only apoptosis inducing pathway 
activated by growth-factor starvation. Indeed, it has been shown that IL-3 activates several pathways involved in the inhibition of apoptosis that are partially redundant. ${ }^{1-3}$ For example, although constitutive activation of the PI3-kinase/ Akt pathway can induce survival of an IL-3 dependent cell line in the absence of growth factor, this pathway is not necessary for the inhibition of apoptosis by IL-3. ${ }^{20}$ Similarly, we have shown in this report and previously ${ }^{20}$ that active glucose metabolism is sufficient to delay apoptosis induced by growth factor withdrawal but is not essential as glycolysis inhibition in the presence of IL-3 does not lead to rapid apoptosis. Our data also suggest that IL-3

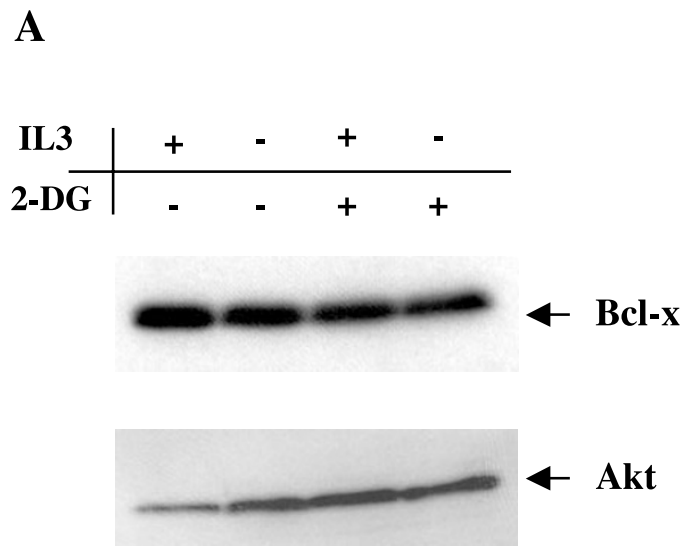

B
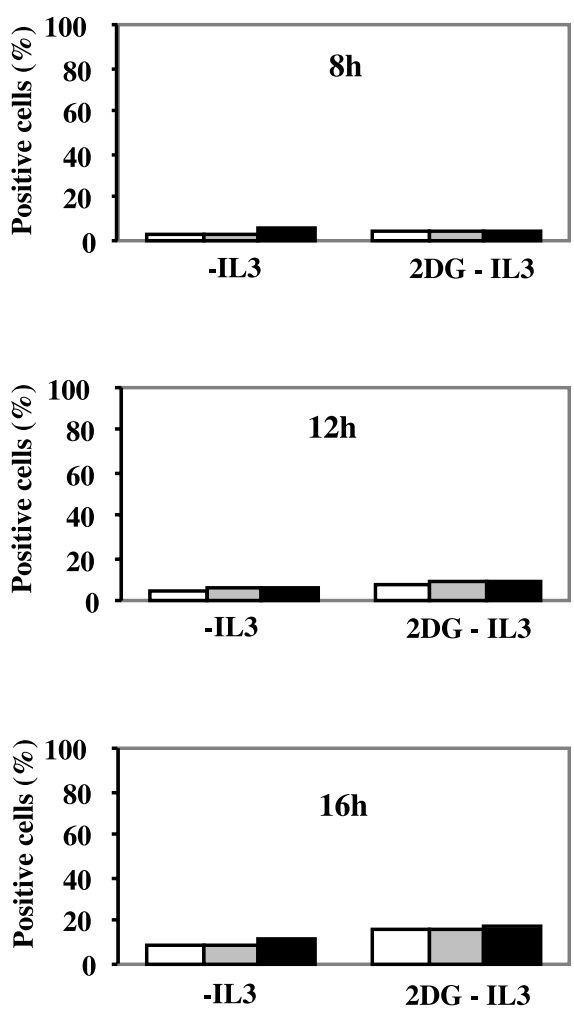

$\mathbf{C}$

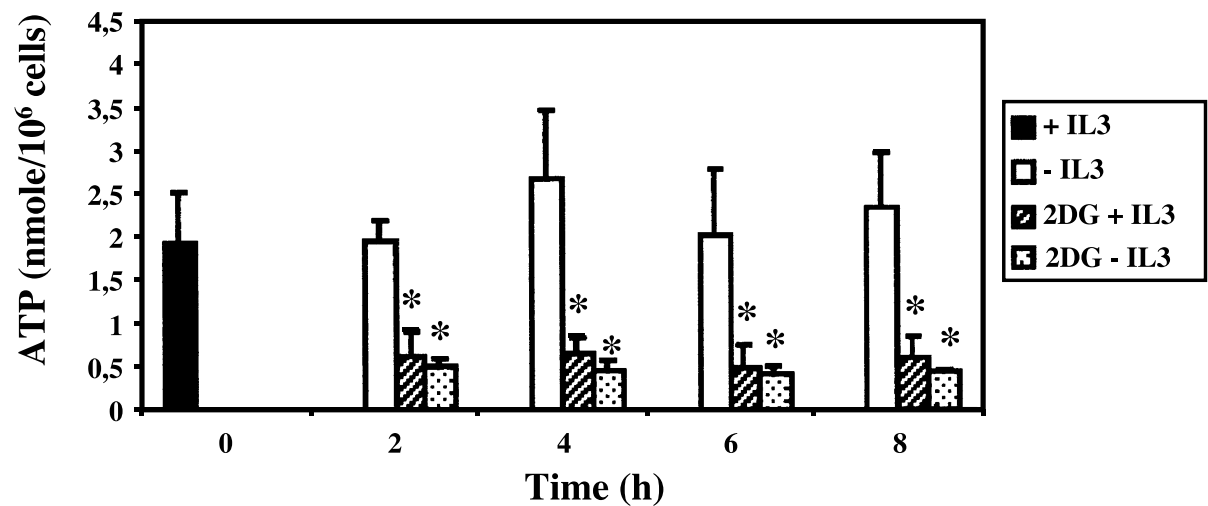

Figure 6 Bcl-X protects Baf-3 cells against glycolysis inhibition. (A) Baf-bcl-x cells were cultured for $8 \mathrm{~h}$ in DMEM or in a low glucose medium containing $6 \mathrm{mM} 2$ deoxy-D-Glucose, in the presence or absence of IL-3. After cell lysis, $50 \mu \mathrm{g}$ of total proteins from each sample were loaded on a 10\% acrylamide gel. Detection of $\mathrm{Bcl}-\mathrm{X}$ and total Akt was done as described in the experimental procedure. (B) Baf-bcl-x cells were cultured in the absence of IL-3 in normal culture medium (-IL3) or low glucose medium containing $6 \mathrm{mM}$ 2-deoxy-D-Glucose (2DG-IL3). Viability was assessed at different time points as indicated on the graphs using Annexin $V$ (white bars), activated Caspase 3 (grey bars) or propidium iodide staining (black bars). One representative experiment out of three is presented. (C) Baf-bcl-x cells were cultured for various time periods in the presence of IL-3 (closed bars), in the absence of IL-3 (empty bars) or in a low glucose medium containing $6 \mathrm{mM} 2$ deoxy-D-Glucose in the presence (hatched bars) or absence (dotted bars) of IL-3. ATP content of Baf-bcl-x cells was determined by Luciferase-Luciferin method. ${ }^{*}: P<0.05$ (two tailed Student's test), at different time points, ATP level values were compared to the one obtained in presence of IL-3 at time 0 
signalling can activate survival pathways that protect against glucose-starvation-induced death. However, inhibition of glycolysis could lead to different outcomes depending on the cellular context. Indeed, it has been reported in clinical studies that 2-deoxy-D-glucose could be used in combination with radiotherapy or chemical treatment to increase the efficacy of therapies. ${ }^{28,29}$ It is thought, that by lowering the cellular energy level, 2-deoxy-Dglucose interferes with the mechanisms involved in the repair of DNA damage thus potentiating these treatments.

In this study we show that IL-3-induced- $b c /-x_{L}$-expression protects cells from apoptosis induced by glucosestarvation. Similar conclusions have been reached by others. Indeed, when cells over-expressing $b c /-x_{L}$ are deprived of IL-3 they survive but undergo a cellular atrophy which is accompanied by a reduction in their glycolytic activity and mitochondrial membrane potential. ${ }^{30,31}$ However, one can not exclude that the increased survival observed in the presence of $\mathrm{IL}-3$ also results from the activation by IL-3 of alternative energy-producing metabolic pathways. In contrast, the metabolism of glucose is essential for the PI3-kinase/Akt survival pathway activated by growth factors. Indeed, the PI3-kinase/Akt pathway is involved in the regulation of the glycolytic metabolism both by up-regulating a number of genes involved in glucose metabolism and by controlling the activity of key glycolytic enzymes. $^{8-10,27,32}$ This action of Akt on the glycolytic metabolism is essential for the prolonged survival observed in the absence of IL-3 in mutants expressing a constitutively active form of Akt. Indeed these mutants cannot survive in the absence of IL-3 in conditions where glycolysis is inhibited as shown in Figure $5 \mathrm{~B}$ and in reference. $^{20}$

Which steps of glycolysis are essential for cell survival? Our results indicate that low levels of ATP alone can not account for the rapid triggering of cell death observed following IL-3 starvation. Since Akt regulates cellular glycolysis at different levels, other metabolites produced by glycolysis or alternatively some glycolytic enzyme regulated by Akt could be involved in the inhibition of apoptosis. For instance, Akt promotes glucose utilisation by controlling the levels and/or the re-localisation of the glucose transporters Glut1, Glut3 and Glut4 and expression of Glut1 can promote survival in the absence of IL-3. ${ }^{8,9,32}$ Akt is also involved in the regulation of mitochondrial physiology as it acts both on glycolysis and oxidative phosphorylation. This process involves the regulation of mitochondria-associated hexokinase activity. ${ }^{13}$ Similarly to Glut1, a link between hexokinase activity and apoptosis inhibition is suggested by experiments showing that constitutive hexokinase expression can overcome the need for Akt activation in the inhibition of apoptosis induced by various stimuli. ${ }^{13}$ As a whole these data suggest that glycolytic activity of cells is strictly monitored and that multiple checkpoints are likely to exist. Any disequilibrium will not only lead to the activation of salvage pathways but also to the triggering of apoptosis before cellular energy levels are too low. ${ }^{33}$

In conclusion, we show that inhibition of glycolysis leads to death by apoptosis and that glycolysis activation is sufficient to protect Baf-3 cells from apoptosis induced by growth factor withdrawal. However, glycolytic metabolism activation is not the main anti-apoptotic pathway activated by IL-3 as its inhibition leads to cell death with slower kinetics than growth factor starvation. We confirmed that constitutive activation of the PI3-kinase/Akt pathway can protect cells from apoptosis in the absence of IL-3 but this protection is dependent on glycolysis activation. This suggests that the ability of Akt to promote survival is correlated to its effects on metabolism stimulation. This is different from apoptosis inhibition induced by $b c l-x_{L}$ overexpression that is independent of glucose uptake and metabolism.

\section{Materials and Methods}

\section{Cell culture}

The bone marrow derived IL-3 dependent Baf-3 cells were grown at $37^{\circ} \mathrm{C}$ in Dulbecco's Modified Eagle's medium (DMEM) (Gibco BRL) containing $6 \%$ foetal calf serum (Boehringer Mannheim), $2 \mathrm{mM} \mathrm{L-}$ glutamine (Gibco BRL) and 5\% WEHI 3B cell-conditioned medium as a source of IL-3. The Baf-3 derived cell lines S4 and Baf-bcl-x that have been described previously were maintained in the same medium. ${ }^{20,23}$ Cells were cultured at a density of $5 \times 10^{4}$ to $8 \times 10^{5}$ cells per ml.

For IL-3 and/or glucose deprivation experiments, cells were washed three times with complete DMEM (4560 mg/l D-glucose) or glucose free DMEM with $6 \%$ FCS and containing or not IL-3. Cells were resuspended in these media. 2-deoxy-D-glucose (Sigma) was used at a final concentration of $6 \mathrm{mM}$.

\section{Cell survival analysis}

Cell viability was detected by propidium iodide (PI) staining and for this purpose, propidium iodide (Sigma) $(5 \mu \mathrm{g} / \mathrm{ml})$ was added to $5 \times 10^{3}$ to $10^{4}$ cells. Cell death analysis was measured by PI or Annexin Staining.

\section{Caspase activities}

For measurement of Caspase 3, 7 and 8 and Caspase 6 and 8 activities, cells $\left(5 \times 10^{6}\right)$ were washed in PBS and lysed for $15 \mathrm{~min}$ at $4^{\circ} \mathrm{C}$ in $100 \mu \mathrm{l}$ lysis buffer ( $250 \mathrm{mM}$ sucrose, $0.1 \%$ Chaps, $10 \mathrm{mM}$ HEPES-KOH pH 7.4, $5 \mathrm{mM}$ DTT, $2 \mathrm{mM}$ EDTA, $20 \mu \mathrm{g} / \mathrm{ml}$ leupeptine, $10 \mu \mathrm{g} / \mathrm{ml}$ pepstatine, $10 \mu \mathrm{g} / \mathrm{ml}$ aprotinine, $1 \mathrm{mM}$ PMSF). After $15 \mathrm{~min}$ centrifugation at $12000 \times g$ and $4^{\circ} \mathrm{C}$, supernatant was recovered to measure caspase activity. Fifty $\mu \mathrm{l}$ of lysis buffer containing $400 \mu \mathrm{M}$ of fluorogenic substrates DEVD-AMC (caspases 3, 7 and 8) or VEIDAMC (caspases 6 and 8 ) were added to $50 \mu \mathrm{l}$ of supernatant and reactions were incubated for $40 \mathrm{~min}$ at $37^{\circ} \mathrm{C}$. Cleavage of the caspase substrates was detected by using a fluoroskan fluorimeter (Labsystems).

For measurement of activated Caspase $3,10^{6}$ cells were fixed and permeabilized with the Cytofix/Cytoperm kit from Pharmingen, following the manufacturer's instructions. The caspase 3 was detected by flow cytometry with a polyclonal rabbit antisera specific for the active form of caspase 3 (Pharmingen, 1/800) and a donkey anti-rabbit antibody coupled to fluorescein isothiocyanate (Immuno research Jackson, 1/50). Staining intensity was recorded using a FACS scan flow cytometer (Beckton Dickinson). 


\section{Lactate production evaluation}

For lactate production measurement, cells were maintained in mediums containing $1 \%$ FCS. For each condition, $2 \times 10^{5}$ cells were centrifuged and lactate concentration in the supernatant was determined using a lactate measurement kit according to the manufacturer's instructions (Sigma Diagnostics). Lactate concentration is expressed in $\mathrm{mmol} / \mathrm{l}$ for $2 \times 10^{5}$ live cells.

\section{ATP concentration determination}

ATP concentration was measured on $5 \times 10^{2}$ live cells by the Luciferase-Luciferin method with an ATP assay kit (Roche Diagnostics) following the manufacturer's instructions. ATP level is expressed in nmole for $10^{6}$ cells.

\section{Northern blot analysis}

Total cellular RNAs were prepared using the RNA Now method (Biogentex). Fifteen $\mu \mathrm{g}$ of total RNA were separated on $1 \%$ agarose formaldehyde gel, transferred to Hybond $\mathrm{N}$ nylon membrane (Amersham Pharmacia Biotech), hybridised overnight at $42^{\circ} \mathrm{C}$ with ${ }^{32} \mathrm{P}$-labelled probes (murine $b \mathrm{cl}-x$ and actin probes) and washed according to standard protocol. Signals were recorded and analyzed with a phosphoimager (Typhoon, Molecular Dynamics).

\section{Gel electrophoresis and immunoblotting}

Cells were harvested and washed in cold PBS before lysis in buffer containing $20 \mathrm{mM}$ Tris pH 7.5, $150 \mathrm{mM} \mathrm{NaCl}, 2 \mathrm{mM}$ EDTA, 1\% NP40, $20 \mu \mathrm{g} / \mathrm{ml}$ aprotinin, $10 \mu \mathrm{g} / \mathrm{ml}$ pepstatine, $20 \mu \mathrm{g} / \mathrm{ml}$ leupeptine, $200 \mu \mathrm{g} /$ $\mathrm{ml} \mathrm{PMSF}$ and $1 \mathrm{mM}$ orthovanadate. For $\mathrm{Bcl}-\mathrm{x}$ analysis, composition of the lysis buffer is $20 \mathrm{mM}$ HEPES pH 7.9, $600 \mathrm{mM} \mathrm{KCl,} 0.2 \mathrm{mM}$ EDTA, $20 \%$ glycerol, $1 \mathrm{mM}$ DTT, anti-protease and anti-phosphatase cocktail. After centrifugation to eliminate cellular debris, protein concentration was determined by the Bradford procedure using the Bio-Rad protein assay. Proteins were separated on $10 \%$ or $12.5 \%$ polyacrylamide SDS-PAGE gels and then electroblotted onto PVDF membranes (Millipore) using a liquid transfer apparatus (Bio-Rad).

For immunoblots, antibodies specific for phospho-Akt or Akt were purchased from New-England Biolabs. Anti-murine Bcl-xL antibody was purchased from Transduction Laboratories. Anti-murine actin antibody (Clone AC-15) was obtained from Sigma. Primary antibodies were detected with either rabbit anti-mouse (for $\mathrm{Bcl}-\mathrm{x}$ and actin) or pig anti-rabbit (for phospho- and total Akt) immunoglobulins conjugated to horseradish peroxidase (DAKO). Immunoblots were revealed with the ECL-kit of Amersham Corp.

\section{Acknowledgements}

S Gonin-Giraud and A-L Mathieu are the recipient of fellowships from La Ligue contre le cancer. This work was supported by institutional grants from the I.N.S.E.R.M. and by specific grants from the Association pour la Recherche contre le Cancer and the Ligue Régionale Rhône-Alpes contre le cancer. We thank Christophe Arpin for statistical analysis and Janet Maryanski and C Arpin for critical reading.

\section{References}

1. LiN, Batzer A, Daly R, Yajnik V, SkolnikE, Chardin P, Bar-Sagi D, Margolis B and Schlessinger J (1993) Guanine-nucleotide-releasing factor hSos 1 binds to Grb2 and links receptor tyrosine kinases to Ras signalling. Nature 363: 85-88
2. Gold MR, Duronio V, Saxena SP, Schrader JW and Aebersold R (1994) Multiple cytokines activate phosphatidylinositol 3-kinase in hemopoietic cells. Association of the enzyme with various tyrosine-phosphorylated proteins. J. Biol. Chem. 269: $5403-5412$

3. Reddy EP, Korapati A, Chaturvedi P and Rane S (2000) IL-3 signaling and the role of Src kinases, JAKs and STATs: a covert liaison unveiled. Oncogene 19: 2532-2547

4. de Peso L, Gonzalez-Garcia M, Page C, Herrera R and Nunez G (1997) Interleukin-3-induced phosphorylation of BAD through the protein kinase Akt. Science 278: $687-689$

5. Songyang Z, Baltimore D, Cantley LC, Kaplan DR and Franke TF (1997) Interleukin 3-dependent survival by the Akt protein kinase. Proc. Natl. Acad. Sci. USA 94: $11345-11350$

6. Brunet A, Bonni A, Zigmond MJ, Lin MZ, Juo P, Hu LS, Anderson MJ, Arden KC, Blenis J and Greenberg ME (1999) Akt promotes cell survival by phosphorylating and inhibiting a Forkhead transcription factor. Cell 96: 857-868

7. Cardone MH, Roy N, Stennicke HR, Salvesen GS, Franke TF, Stanbridge E, Frisch S and Reed JC (1998) Regulation of cell death protease caspase-9 by phosphorylation. Science 282: 1318-1321

8. Barthel A, Okino ST, Liao J, Nakatani K, Li J, Whitlock Jr JP and Roth RA (1999) Regulation of GLUT1 gene transcription by the serine/threonine kinase Akt1. J. Biol. Chem. 274: 20281-20286

9. Kohn AD, Summers SA, Birnbaum MJ and Roth RA (1996) Expression of a constitutively active Akt Ser/Thr kinase in 3T3-L1 adipocytes stimulates glucose uptake and glucose transporter 4 translocation. J. Biol. Chem. 271: 3137231378

10. Cross DA, Alessi DR, Cohen P, Andjelkovich M and Hemmings BA (1995) Inhibition of glycogen synthase kinase-3 by insulin mediated by protein kinase $B$. Nature 378: $785-789$

11. Pap M and Cooper GM (1998) Role of glycogen synthase kinase-3 in the phosphatidylinositol 3-Kinase/Akt cell survival pathway. J. Biol. Chem. 273: $19929-19932$

12. Crowder RJ and Freeman RS (2000) Glycogen synthase kinase-3 beta activity is critical for neuronal death caused by inhibiting phosphatidylinositol 3-kinase or Akt but not for death caused by nerve growth factor withdrawal. J. Biol. Chem. 275: 34266-34271

13. Gottlob K, Majewski N, Kennedy S, Kandel E, Robey RB and Hay N (2001) Inhibition of early apoptotic events by Akt/PKB is dependent on the first committed step of glycolysis and mitochondrial hexokinase. Genes Dev. 15: $1406-1418$

14. Kan O, Baldwin SA and Whetton AD (1994) Apoptosis is regulated by the rate of glucose transport in an interleukin 3 dependent cell line. J. Exp. Med. 180: 917 923

15. Dang CV and Semenza GL (1999) Oncogenic alterations of metabolism. Trends Biochem. Sci. 24: 68-72

16. Rathmell JC, Vander Heiden MG, Harris MH, Frauwirth KA and Thompson CB (2000) In the absence of extrinsic signals, nutrient utilization by lymphocytes is insufficient to maintain either cell size or viability. Mol. Cell 6: 683-692

17. Vander Heiden MG, Plas DR, Rathmell JC, Fox CJ, Harris MH and Thompson CB (2001) Growth factors can influence cell growth and survival through effects on glucose metabolism. Mol. Cell. Biol. 21: 5899-5912

18. Palacios R and Garland J (1984) Distinct mechanisms may account for the growth-promoting activity of interleukin 3 on cells of lymphoid and myeloid origin. Proc. Natl. Acad. Sci. USA 81: 1208-1211

19. Garland JM and Halestrap A (1997) Energy metabolism during apoptosis. Bcl-2 promotes survival in hematopoietic cells induced to apoptose by growth factor withdrawal by stabilizing a form of metabolic arrest. J. Biol. Chem. 272: 46804688

20. Mathieu AL, Gonin S, Leverrier Y, Blanquier B, Thomas J, Dantin C, Martin G, Baverel Gand MarvelJ (2001) Activation of the phosphatidylinositol3-kinase/Akt pathway protects against interleukin-3 starvation but not DNA damage-induced apoptosis. J. Biol. Chem. 276: 10935-10942

21. LeverrierY, Thomas J, Mathieu AL, Low W, BlanquierB and MarvelJ (1999) Role of PI3-kinase in Bcl-X induction and apoptosis inhibition mediated by IL-3 or IGF1 in Baf-3 cells. Cell Death Differ. 6: 290-296

22. Leverrier $Y$, Thomas J, Perkins GR, Mangeney M, Collins MK and Marvel J (1997) In bone marrow derived Baf-3 cells, inhibition of apoptosis by IL-3 is mediated by two independent pathways. Oncogene 14: 425-430 
23. Thomas J, Leverrier $\mathrm{Y}$ and Marvel $\mathrm{J}$ (1998) Bcl- $\mathrm{X}$ is the major pleiotropic antiapoptotic geneactivated by retroviral insertion mutagenesis in an IL-3 dependent bone marrow derived cell line. Oncogene 16: 1399-1408

24. Khaled AR, Reynolds DA, Young HA, Thompson CB, Muegge K and Durum SK (2001) Interleukin-3 withdrawal induces an early increase in mitochondrial membrane potential unrelated to the $\mathrm{Bcl}-2$ family. Roles of intracellular $\mathrm{pH}, \mathrm{ADP}$ transport, and F(0)F(1)-ATPase. J. Biol. Chem. 276: 6453-6462

25. Whetton AD, Bazill GW and Dexter TM (1984) Haemopoietic cell growth factor mediates cell survival via its action on glucose transport. EMBO J. 3: 409-413

26. Ahmed N and Berridge MV (1997) Regulation of glucose transport by interleukin3 in growth factor-dependent and oncogene-transformed bone marrow-derived cell lines. Leuk. Res. 21: 609-618

27. Deprez J, Vertommen D, Alessi DR, Hue L and RiderMH (1997)Phosphorylation and activation of heart 6-phosphofructo-2-kinase by protein kinase $B$ and other protein kinases of the insulin signaling cascades. J. Biol. Chem. 272: 1726917275

28. Halicka HD, Ardelt B, Li X, Melamed MM and Darzynkiewicz Z (1995) 2-DeaoxyD-glucose enhances sensitivity of human histiocytic lymphoma U937 cells to apoptosis induced by tumor necrosis factor. Cancer Res. 55: 444-449
29. Mohanti BK, Rath GK, Anantha N, Kannan V, Das BS, Chandramouli BA Banerjee AK, Das S, Jena A, Ravichandran R, Sahi UP, Kumar R, Kapoor N Kalia VK, Dwarakanath BS and Jain V (1996) Improving cancer radiotherapy with 2-deoxy-D-glucose: phase I/II clinical trials on human cerebral gliomas. Int. J. Radiat. Oncol. Biol. Phys. 35: 103-111

30. Plas DR, Talapatra S, Edinger AL, Rathmell JC and Thompson CB (2001) Akt and $\mathrm{Bcl}-\mathrm{xL}$ promote growth factor-independent survival through distinct effects on mitochondrial physiology. J. Biol. Chem. 276: 12041-12048

31. VanderHeiden MG, Chandel NS, SchumackerPT and Thompson CB (1999) Bcl$\mathrm{XL}$ prevents cell death following growth factor withdrawal by facilitating mitochondrial ATP/ADP exchange. Mol. Cell 3: 159-167

32. Hajduch E, Alessi DR, Hemmings BA and Hundal HS (1998) Constitutive activation of protein kinase $B$ alpha by membrane targeting promotes glucose and system A amino acid transport, protein synthesis, and inactivation of glycogen synthase kinase 3 in L6 muscle cells. Diabetes 47: 1006-1013

33. Faradji RN, Havari E, Chen Q, Gray J, Tornheim K, Corkey BE, Mulligan RC and Lipes MA (2001) Glucose-induced toxicity in insulin-producing pituitary cells that coexpress GLUT2 and glucokinase. Implications for metabolic engineering. J. Biol. Chem. 276: 36695-36702 